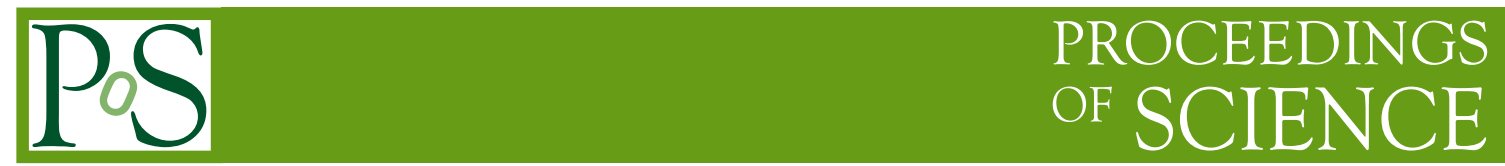

\title{
Recent Results on Radiative and Electroweak Penguin B Decays at Belle
}

\author{
Akimasa Ishikawa* \\ Tohoku University \\ E-mail: akimasadepx.phys.tohoku.ac.ip
}

We report on recent results on radiative and electroweak penguin $B$ decays at Belle at the KEKB collider.

XIII International Conference on Heavy Quarks and Leptons

22-27 May, 2016

Blacksburg, Virginia, USA

\footnotetext{
* Speaker.
} 


\section{Introduction}

$B$ meson decays via loop diagrams are sensitive to physics beyond the Standard Model (BSM) since (1) the processes in the SM are suppressed by the Cabibbo-Kobayashi-Maskawa (CKM) matrix elements, $V_{t s}$ or $V_{t d}$, and loop factor, and (2) unobserved heavy particles might be able to enter in the loop with comparable amplitudes. Radiative and electroweak penguin decays are experimentally and theoretically clean due to final states having color singlet leptons or photons. Thus these are ideal tools to search for BSM.

For the radiative and electroweak analyses described below, we used full data sample of $711 \mathrm{fb}^{-1}$ accumulated by the Belle detector at the KEKB energy-asymmetric collider.

\section{Measurement of $B \rightarrow X_{s} \gamma$ with Sum-of-Exclusive Method}

The branching fraction (BF) of inclusive $b \rightarrow s \gamma$ is very sensitive to BSM, such as supersymmetry or charged Higgs. The BF is precisely predicted in the SM [四], $\mathscr{B}\left(B \rightarrow X_{s} \gamma\right)=(3.36 \pm$ $0.23) \times 10^{-4}$, and world averages of experiments by HFAG or PDG [ [ [ ] ] are consistent with the predictions. To improve the sensitivity to BSM, both experiment and theory should reduce the error.

We measured the BF of $B \rightarrow X_{s} \gamma$ with a sum-of-exclusive method using the highest ever statistics which allows to reduce the dominant systematics due to fragmentation of $X_{s}$ system. We reconstructed $38 X_{s}$ modes, $K \pi, K 2 \pi, K 3 \pi K 4 \pi, K \eta(\eta \rightarrow \gamma \gamma), K \eta \pi, 3 K$ and $3 K \pi$, in which at most two neutral pions and one $K_{S}^{0}$ are allowed. The mass of the hadronic system was required to be less than $2.8 \mathrm{GeV} / c^{2}$, which corresponds to a photon energy threshold of $1.9 \mathrm{GeV}$, to suppress a large combinatorial background from $B \bar{B}$ events. Continuum background was suppressed based on neural net with event shape variables.

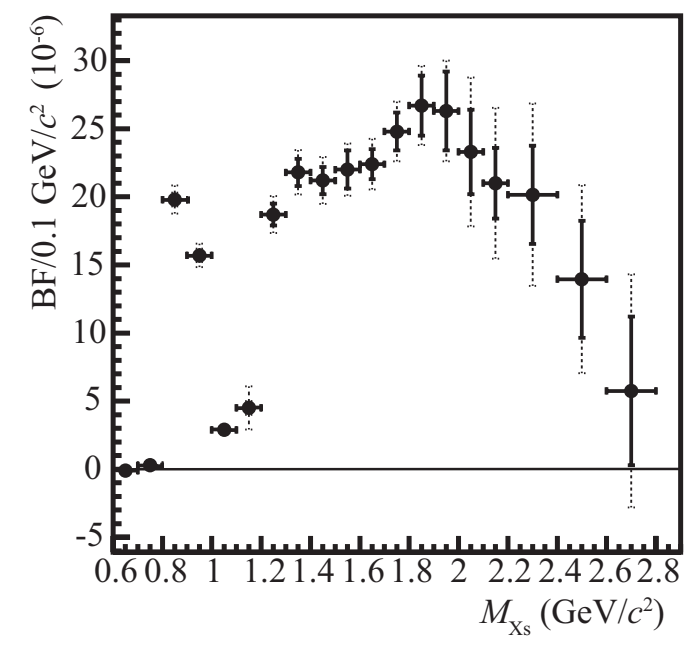

Figure 1: Measured partial BF as a function of $M_{X_{S}}$. Solid and dotted lines are statistical and total errors.

Signal yield for each $M_{X_{s}}$ bin is obtained by fitting to the beam energy constrained mass $\left(M_{b c}\right)$ distribution defined as $M_{b c}=\sqrt{E_{\text {beam }}^{2}-p_{B}^{2}}$, where $E_{\text {beam }}$ is the beam energy and $p_{B}$ is the measured 
$B$ meson momentum in the center of mass system. The measured partial BF is shown in Fig. $\mathbb{\text { m. }}$ To study the fragmentation of $X_{s}$ system in data, we also extracted signal yields for subsets of final states in $M_{X_{s}}$ bins which were used for calibration of PYTHIA parameters [ [ $\forall$ ].

Finally, we measured the BF extrapolated to a photon energy threshold of $1.6 \mathrm{GeV}$ in order to compare with theoretical predictions, of $\mathscr{B}\left(B \rightarrow X_{s} \gamma\right)=(3.75 \pm 0.18 \pm 0.35) \times 10^{-4}$, where the first error is statistical and the second is systematic [[]]. This result is the most sensitive measurement using the sum-of-exclusive method (Fig. $\square$ ). Using the world average by PDG, we set the limit of charged Higgs in the two Higgs doublet model. Since $\tan \beta$ and $\cot \beta$ in the dominant contribution from $b-t-H$ and $t-s-H$ vertices cancel out if $\tan \beta$ is not too small, the charged Higgs contribution is almost independent on $\tan \beta$ value. We set the limit on charged Higgs mass as $M_{H^{+}}>480 \mathrm{GeV} / c^{2}$ at $95 \%$ C.L.

Recently, we also measured the BF with fully inclusive photon analysis and the preliminary result is the world most precise measurement [䧃].

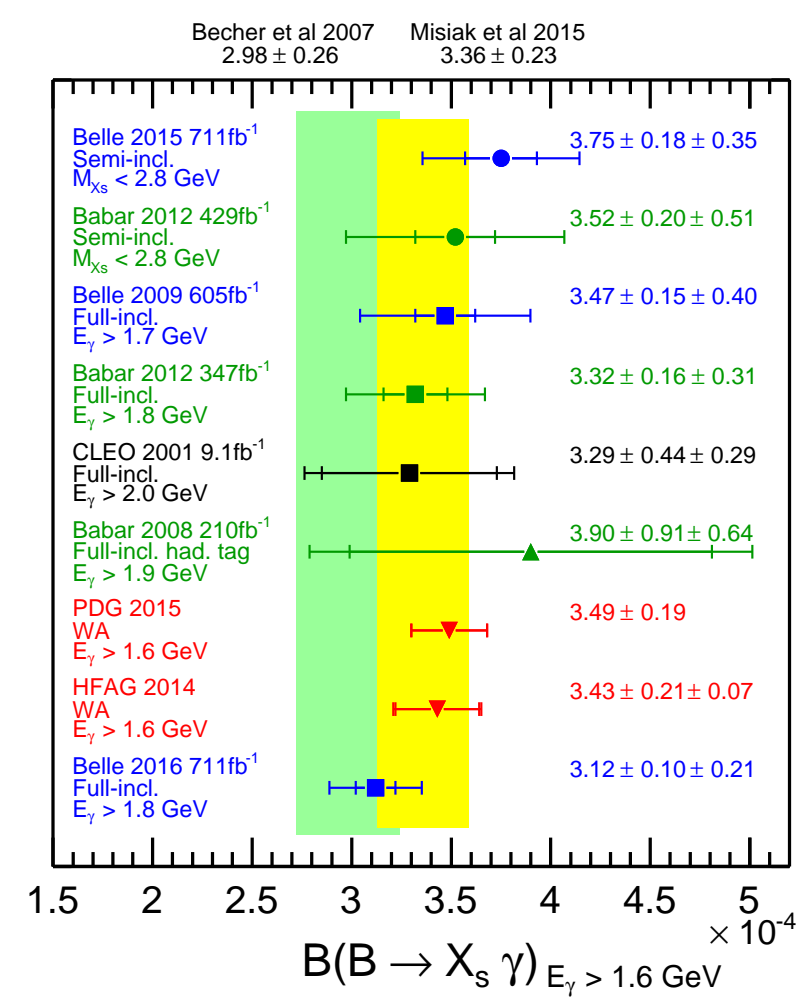

Figure 2: Summary of measured branching fraction of $B \rightarrow X_{s} \gamma$ compared with theoretical predictions [四, 四].

\section{Measurement of Direct $C P$ Violation in $B \rightarrow X_{s+d} \gamma$}

Recent theoretical studies show that uncertainty of direct $C P$ violation $(C P V)$ in $b \rightarrow s \gamma$ is about $\mathscr{O}(2 \%)[[]$, which is larger than prior expectation [Q] due to newly accounted resolved photon uncertainty. However thanks to U-spin relations and unitarity of the CKM matrix, direct $C P V$ 
of combined $b \rightarrow s \gamma$ and $b \rightarrow d \gamma$ (denoted as $b \rightarrow s+d \gamma$ in this manuscript) is very small [Q]. If the measured $C P V$ deviates from null, it is a clear BSM signal. We first reconstructed hard photons with loose energy selection of $1.7 \mathrm{GeV}$ to $2.8 \mathrm{GeV}$. Large backgrounds from asymmetric $\pi^{0}$ and $\eta$ decays were vetoed by the invariant mass with another photon. To reduce the continuum background, high momentum lepton was required. In the signal events, this lepton should come from the other $B$ meson thus the flavor of the signal can be tagged by the charge of the lepton. Dilutions due to mixing in the $B^{0} \overline{B^{0}}$ events and secondary lepton was corrected. Fig. B B shows the photon spectra of the background-subtracted signal tagged with positively and negatively charged leptons. To maximize the sensitivity, the photon energy is required to be greater than $2.1 \mathrm{GeV}$. The result is $\mathscr{A}_{C P}\left(B \rightarrow X_{s+d} \gamma\right)=(2.2 \pm 4.0 \pm 0.8) \%$ [ए]] which is world best measurement and even better than the average by PDG in 2015 (Fig.(1) [3].

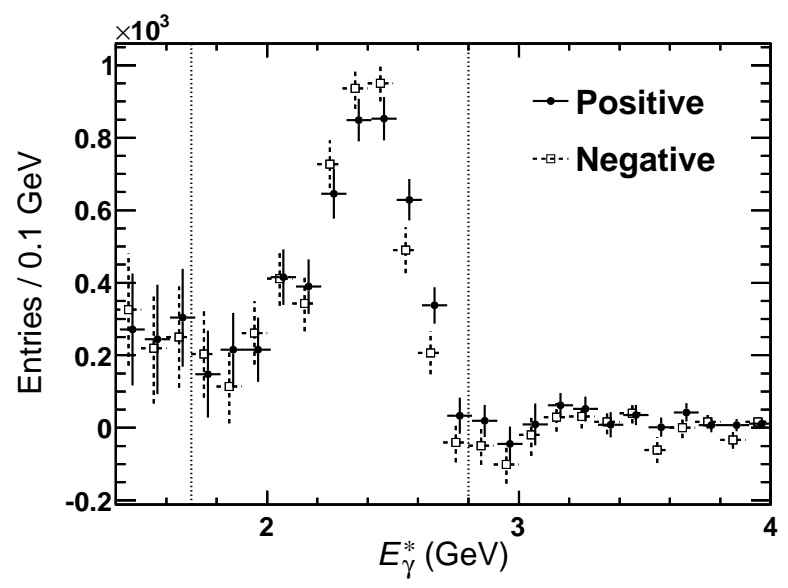

Figure 3: Photon spectra tagged with positively and negatively charged lepton.

\section{Search for $B \rightarrow \phi \gamma$}

The $B \rightarrow \phi \gamma$ decay proceeds through a penguin annihilation diagram which is suppressed by the CKM matrix element $V_{t d}$. The BF in the SM is predicted as $\mathscr{O}\left(10^{-12}\right)$ to $\mathscr{O}\left(10^{-11}\right)$ [四] which is not accessible at Belle. However, BSM enhances the BF to $\mathscr{O}\left(10^{-9}\right)$ to $\mathscr{O}\left(10^{-8}\right)$. We searched for the decay using the $\phi \rightarrow K^{+} K^{-}$sub-decay mode which is very clean thanks to the small width and $\mathrm{Q}$ value. The signal events are extracted by a four-dimensional fit with $M_{b c}, \Delta E$, neural net output, and helicity angle of the $\phi \rightarrow K^{+} K^{-}$decay. Fig 1 shows projections onto $M_{b c}$ and $\Delta E$ distributions. The result is consistent with null and an upper limit on the BF was set as $\mathscr{B}(B \rightarrow \phi \gamma)<1.0 \times 10^{-7}$ [ㅁ] which is just one order of magnitude higher than predictions within some new physics models.

\section{Full Angular Analysis of $B \rightarrow K^{*} \ell^{+} \ell^{-}$}

The $b \rightarrow s \ell^{+} \ell^{-}$decays were observed by Belle Collaboration about 15 years ago [एँ3] which opened new door to search for BSM. The BF and Forward-Backward Asymmetry (AFB) as func- 


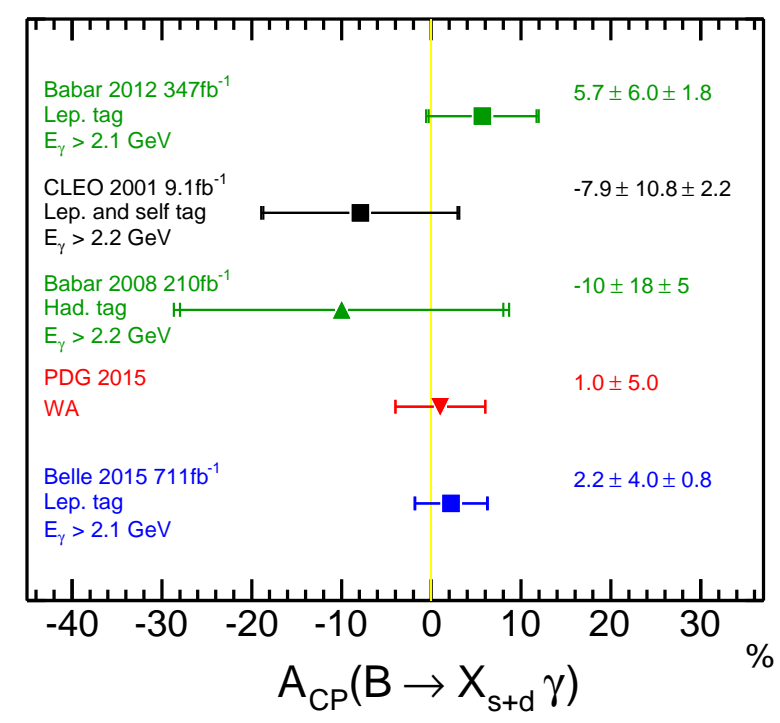

Figure 4: Summary of measured direct $C P V$ in $B \rightarrow X_{s+d} \gamma$.
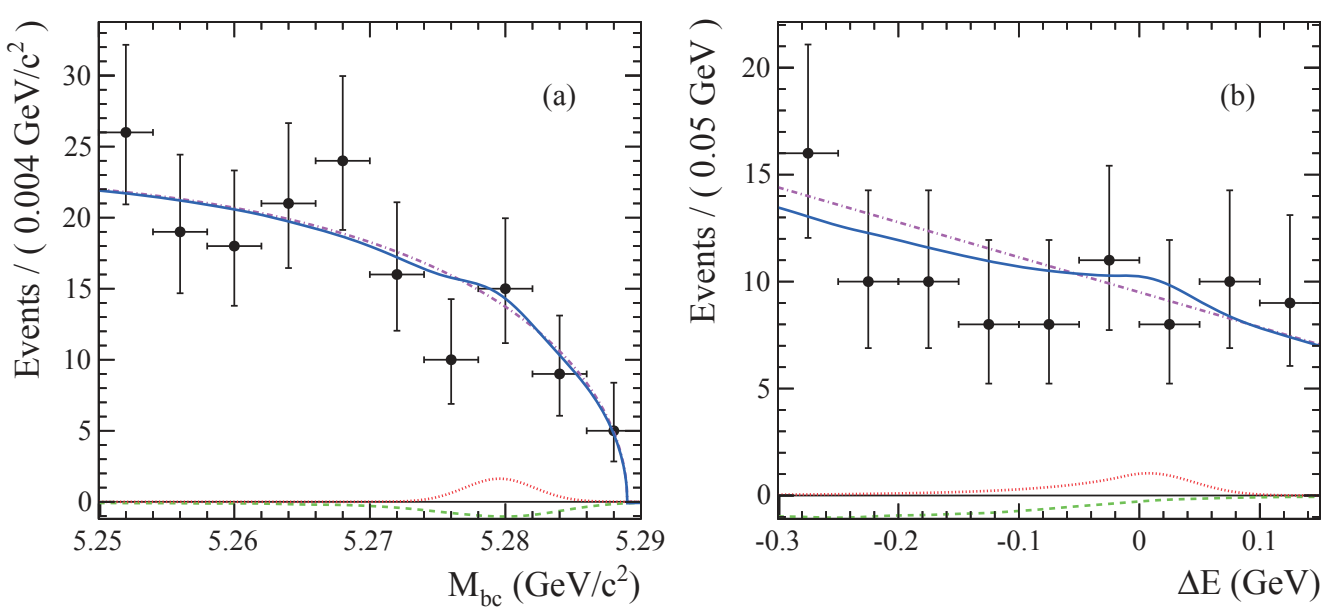

Figure 5: $M_{b c}$ and $\Delta E$ distributions for $B \rightarrow \phi \gamma$.

tions of $q^{2}$ in $B \rightarrow K^{*} \ell^{+} \ell^{-}$are important observables for BSM searches, and several experiments already measured these [14]. Full angular analysis of $B \rightarrow K^{*} \ell^{+} \ell^{-}$with optimized observables [प5], which are insensitive to form factor uncertainties, are very powerful tools to search for BSM. LHCb first reported the results [[6] and one of the observables, $P_{5}^{\prime}$, deviates by about $3.4 \sigma$ from a prediction of the SM by DHMV [ㅁ] ]. (There is a discussion in theory community that the deviation might be able to be explained by charm-loop [지, [1, [2]].) By a global fit to observables in $b \rightarrow s \gamma$ and $b \rightarrow s \ell^{+} \ell^{-}$including $P_{5}^{\prime}$, one of the Wilson coefficients, $C_{9}$, deviates by about $-30 \%$ from the $\mathrm{SM}$ prediction [2]]. This could indicate BSM in the $b \rightarrow s \ell^{+} \ell^{-}$process. 
We also measured the optimized observables using $B^{0} \rightarrow K^{* 0} \ell^{+} \ell^{-}$, where the $\ell$ stands for electron or muon. Even with full data, we expected only 200 signal events which is about 10 times smaller than that at $\mathrm{LHCb}$, and hence the selection criteria should be optimized better than the previous analysis. We adopted neural net based analysis to select signal candidates and to suppress backgrounds. Signal is extracted by fitting to $M_{\mathrm{bc}}$ distributions. We observed $69 \pm 11$ and $118 \pm 12$ signal events for the electron and muon modes, respectively. For the full angular analysis, we adopted the folding method on angular variables, $\theta_{\ell}, \theta_{K}$ and $\phi$, to extract the optimized observables which LHCb performed in 2013. The fit results for $P_{5}^{\prime}$ are shown in Fig.6 [22]. The result for $4<q^{2}<8$ is about $2.1 \sigma$ deviation from the prediction by DHMV [प]] and is consistent with $\mathrm{LHCb}$ result [16]. We also compared the results with other theoretical predictions of the SM [18, [9] and the tendency of the deviation for $P_{5}^{\prime}$ is the same. The other optimized observables, $P_{4}^{\prime}, P_{6}^{\prime}$ and $P_{8}^{\prime}$, are consistent with the predictions within errors. By combining with LHCb result, the deviation of $P_{5}^{\prime}$ from a prediction by DHMV is about $4 \sigma$.

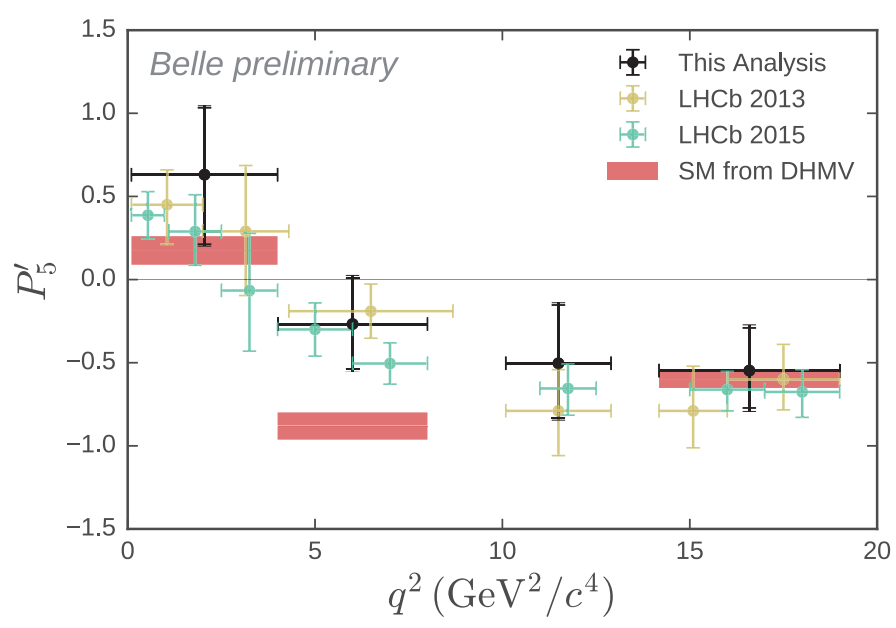

Figure 6: Comparison of $P_{5}^{\prime}$ distributions in $B^{0} \rightarrow K^{* 0} \ell^{+} \ell^{-}$.

\section{Measurement of Forward-Backward Asymmetry in $B \rightarrow X_{s} \ell^{+} \ell^{-}$}

AFB in $B \rightarrow K^{*} \ell^{+} \ell^{-}$was first measured by Belle [23] and then done by several experiments, while the AFB in the inclusive process $B \rightarrow X_{s} \ell^{+} \ell^{-}$, which is much more cleanly predicted in the SM [24] than exclusive decays, was not yet measured. Since current global fit shows deviation in $C_{9}$, a measurement of AFB in $B \rightarrow X_{5} \ell^{+} \ell^{-}$provides an independent check of the deviation of the Wilson coefficient from expectation. Belle has performed the first measurement of the AFB in $B \rightarrow X_{s} \ell^{+} \ell^{-}$with the sum-of-exclusive technique. We reconstructed 36 decay modes, of which 20 self-tag modes are used to measure AFB. To reduce the backgrounds from continuum and $B \bar{B}$ events, we used a neural net with event shape variables, vertex quality, and flavor tagging quality. Since combinatorial backgrounds are large, we must require the invariant mass of $X_{s}$ system to be less than $2.0 \mathrm{GeV} / c^{2}$. To extract the signal events, fits to $M_{\mathrm{bc}}$ distributions for forward and 
backward events were performed. The AFB is calculated from the signal events with correction factors obtained from Monte Carlo samples which are calibrated with real data. Fig. $\square$ shows the results for AFB [D2]] as a function of $q^{2}$ which is consistent with a theoretical prediction [[26].

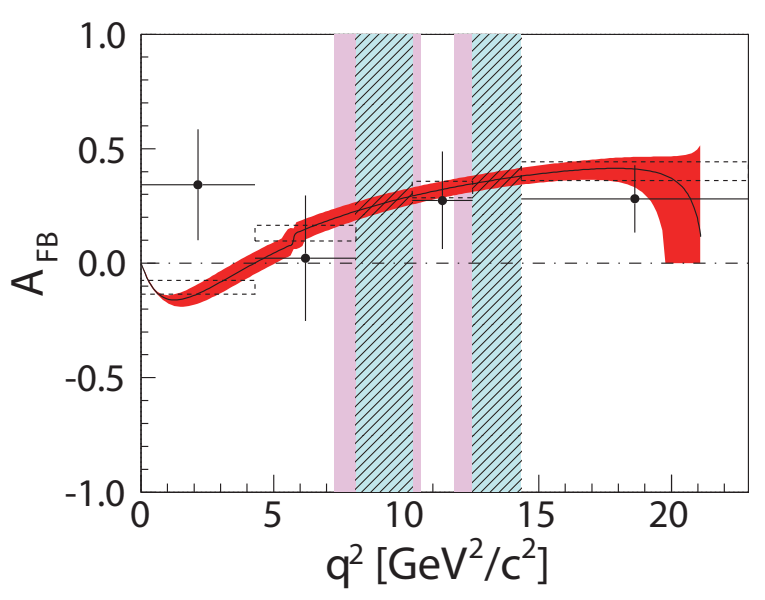

Figure 7: Measured AFB in $B \rightarrow X_{s} \ell^{+} \ell^{-}$compared with a theoretical prediction [26].

\section{Search for $B \rightarrow h v \bar{v}$}

The di-neutrino emission process, $B \rightarrow h v \bar{v}$, is not observed yet [27]. This loop process is theoretically interesting since clean prediction is possible thanks to the lack of contributions from charm-loop diagrams [28], and BSM effects, such as $C_{9}$ deviation, could be correlated with $b \rightarrow s \ell^{+} \ell^{-}$in some models.

We searched for the $B \rightarrow h v \bar{v}$ decays, where hadronic systems are $\pi^{0}, \pi^{+}, K_{S}^{0}, K^{+}, \rho^{0}, \rho^{+}$, $K^{* 0}, K^{*+}$ or $\phi$. Since two neutrinos are in the final states, the other $B$ mesons should be tagged. We reconstructed 1104 exclusive hadronic $B$ decays as the tagging side whose efficiencies are about $0.3 \%$ and $0.2 \%$ for $B^{+}$and $B^{0}$, respectively. Then, we required momentum of $h$ candidates greater than $1.6 \mathrm{GeV} / c$. We chose extra energy in electromagnetic caloriemeter as the final discriminator as shown in Fig. [ , and found the distribution is consistent with background. We set upper limits on the decays ranging $(4-21) \times 10^{-5}$, and obtained the world best limits for $K^{*+}, \pi^{+}, \pi^{0}, \rho^{+}$[29]. The limits on BFs for $K^{*}$ modes are just 5 times larger than theoretical predictions in the SM [28], and thus Belle II can observe these decay modes.

\section{Summary}

We have studies radiative and electroweak penguin processes with the full data set at the Belle experiment. The $P_{5}^{\prime}$ observable measured with the full angular analysis of $B^{0} \rightarrow K^{* 0} \ell^{+} \ell^{-}$deviates by about $2.1 \sigma$ from the SM prediction. This could be further studied using charged $B$ meson decays and measurements of lepton universality for the optimized observables. Other results are consistent with SM predictions, thus strong limits on BSM models are set. 


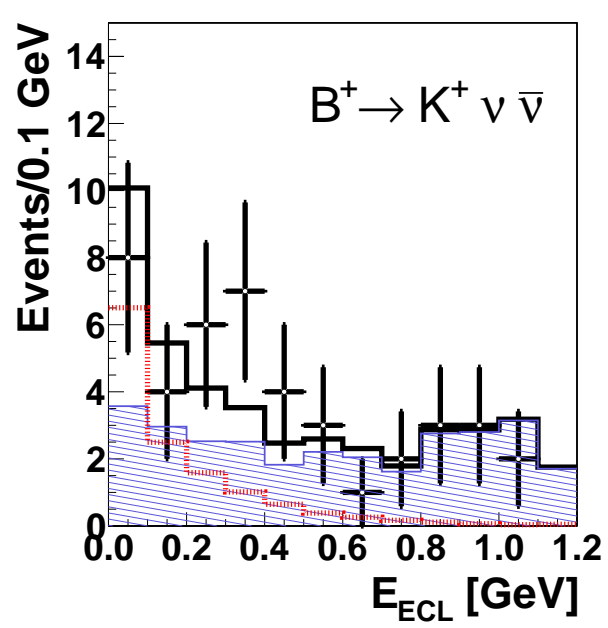

Figure 8: $E_{\mathrm{ECL}}$ distribution for $B \rightarrow K^{+} v \bar{v}$.

\section{Acknowledgments}

A. Ishikawa is supported by a Grant-in-Aid from JSPS for Scientific Research (B) No. 16H03968.

\section{References}

[1] M. Misiak et al., Phys. Rev. Lett. 114, 221801 (2015).

[2] http://www.slac.stanford.edu/xorg/hfag/.

[3] K. A. Olive et al. (Particle Data Group), Chin. Phys. C 38, 090001 (2014) and 2015 update.

[4] T. Sjostrand, S. Mrenna and P. Skands, J. High Energy Phys. 0605, 026 (2006).

[5] T. Saito, A. Ishikawa, et al. (Belle Collaboration), Phys. Rev. D 91, 052004 (2015).

[6] A. Abdesselam, et al. (Belle Collaboration), arXiv:1608.02344.

[7] T. Becher and M. Neubert, Phys. Rev. Lett. 98, 022003 (2007).

[8] M. Benzke, S. J. Lee, M. Neubert and G. Paz, Phys. Rev. Lett. 106, 141801 (2011).

[9] T. Hurth, E. Lunghi and W. Porod, Nucl. Phys. B 704, 56 (2005).

[10] L. Pesantez, et al. (Belle Collaboration), Phys. Rev. Lett. 114, 151601 (2015).

[11] X.-Q. Li, G.-R. Lu, R.-M. Wang, and Y. Yang, Eur. Phys. J. C 36, 97 (2004); J. Hua, C. Kim, and Y. Li, Eur. Phys. J. C 69, 139 (2010), C.-D. Lu, Y.-L. Shen, and W. Wang, Chin. Phys. Lett. 23, 2684 (2006).

[12] Z. King, et al. (Belle Collaboration), Phys. Rev. D 93, 111101 (2016).

[13] K. Abe, A. Ishikawa, et al. (Belle Collaboration), Phys. Rev. Lett. 88, 021801 (2002); J. Kaneko, et al. (Belle Collaboration), Phys. Rev. Lett. 90, 021801 (2003); A. Ishikawa, et al. (Belle Collaboration), Phys. Rev. Lett. 91, 261601 (2003). 
[14] R. Aaji, et al. (LHCb Collaboration), arXiv:1606.04731, submitted to J. High Energy Phys.; R. Aaji, et al. (LHCb Collaboration), J. High Energy Phys. 1602, 104 (2016); J. P. Lees, et al. (Babar Collaboration), Phys. Rev. D 93, 052015 (2016); V. Khachatryan, et al. (CMS Collaboration), Phys. Lett. B 753, 424 (2016); T. Aaltonen, et al. (CDF Collaboration), Phys. Rev. Lett. 108, 081807 (2012); J. P. Lees, et al. (Babar Collaboration), Phys. Rev. D 86, 2012 (032012); J.-T. Wei, et al. (Belle Collaboration), Phys. Rev. Lett. 103, 171801 (2009).

[15] S. Descotes-Genon, T. Hurth, J. Matias and J. Virto, J. High Energy Phys. 1305, 2013 (137).

[16] R. Aaji, et al. (LHCb Collaboration), J. High Energy Phys. 1602, 104 (2016); R. Aaji, et al. (LHCb Collaboration), J. High Energy Phys. 1308, 131 (2013).

[17] S. Descotes-Genon, L. Hofer, J. Matias and J. Virto, J. High Energy Phys. 1412, 125 (2014).

[18] S. Jäger and J. M. Camalich, Phys. Rev. D 93, 014028 (2016); S. Jäger and J. M. Camalich, J. High Energy Phys. 1305, 043 (2013).

[19] A. Bharucha, D. M. Straub and R. Zwicky, J. High Energy Phys. 1608, 98 (2016).

[20] M. Ciuchini, et al., J. High Energy Phys. 1606, 116 (2016); J. Lyon and R. Zwicky, arXiv:1406.0566; A. Khodjamirian, Th. Mannel, A. A. Pivovarov and Y.-M. Wang, J. High Energy Phys. 1009, 089 (2010).

[21] See for example, S. Descotes-Genon, J. Matias and J. Virto, Phys. Rev. D 88, 074002 (2013); W. Altmannshofer and D. M. Straub, arXiv:1503.06199.

[22] A. Abdesselam, et al. (Belle Collaboration), arXiv:1604.04042.

[23] A. Ishikawa, et al. (Belle Collaboration), Phys. Rev. Lett. 96, 251801 (2006).

[24] T. Huber, T. Hurth and E. Lunghi, J. High Energy Phys. 1506, 176 (2015).

[25] Y. Sato, A. Ishikawa, et al. (Belle Collaboration), Phys. Rev. D 93, 032008 (2016).

[26] S. Fukae, C. S. Kim, T. Morozumi and T. Yoshikawa, Phys. Rev. D 59, 074013 (1999).

[27] J. P. Lee, et al. (Babar Collaboration), Phys. Rev. D 87, 112005 (2013).

[28] A. J. Buras, J. Girrbach-Noe, C. Niehoff and D. M. Straub, J. High Energy Phys. 1502, 184 (2015).

[29] O. Lutz, et al. (Belle Collaboration), Phys. Rev. D 87, 111103(R) (2013). 